\title{
AVALIAÇÃO DE COBERTURAS COMESTÍVEIS PARA CONSERVAÇÃO DE PIMENTA-BIQUINHO (Capsicum chinense JACQ.)
}

\author{
Graziela Silva Oliveira ${ }^{1}$, Nataly de Almeida Costa ${ }^{1}$, Cleide Maria Ferreira Pinto ${ }^{2}$, Cláudia Lúcia de \\ Oliveira Pinto ${ }^{3}$, Sérgio Mauricio Lopes Donzeles ${ }^{4}$, Eliane Maurício Furtado Martins ${ }^{5}$
}

\begin{abstract}
RESUMO - A demanda pela pimenta-biquinho Capsicum chinense Jacq. é crescente sendo um dos problemas na sua cadeia produtiva a perda de massa e de características típicas do fruto. Assim, o estudo de alternativas sobre a conservação pós-colheita tem grande importância para produtores, processadores e consumidores. Desta forma, objetivou-se avaliar o uso de coberturas comestíveis para a conservação de frutos pimenta-biquinho. Frutos lavados e sanitizados foram imersos em coberturas à base de cera de carnaúba, fécula de mandioca, gelatina e pectina cítrica e armazenados à temperatura ambiente de $25^{\circ} \mathrm{C}$. Frutos lavados e, apenas sanitizados, constituíram um dos tratamentos e, frutos apenas lavados constituíram o controle. No tempo zero e a cada 3 dias, por 12 dias, realizaram-se análises físico-químicas, microbiológicas, e avaliação visual dos frutos. Não houve diferença $(\mathrm{p}>0,05)$ para $\mathrm{pH}$, acidez titulável e teor de sólidos solúveis entre tratamentos, entretanto houve diferença $(p<0,05)$ com relação ao tempo de armazenamento. Para firmeza não houve diferença $(p>0,05)$ entre tratamentos. A coloração vermelha ou amarela dos frutos manteve-se estável durante o armazenamento, independente do tratamento. $\mathrm{O}$ índice Chroma variou $(\mathrm{p}<0,05)$ com o tempo de armazenamento. Em todos os tratamentos, observou-se perda de massa ao longo do tempo de estocagem. Concluiu-se que as características físico-químicas como $\mathrm{pH}$, acidez titulável, teor de sólidos solúveis, firmeza e coloração não são alteradas com utilização das coberturas comestíveis. Entretanto, estas mesmas coberturas não contribuem para minimizar a perda de massa dos frutos com consequente ocorrência de enrugamento e escurecimento ao final de 12 dias de armazenamento. A cobertura à base de fécula de mandioca $3 \%$ permite manter as características visuais da pimenta-biquinho por 12 dias. A retirada do pedúnculo pode ter contribuído para o aumento da contaminação microbiana dos frutos.
\end{abstract}

Palavras chave: biofilmes, Capsicum spp., pós-colheita, revestimento comestivel, vida de prateleira.

\section{EVALUATION OF EDIBLE COVERINGS FOR THE CONSERVATION OF BIQUINHO PEPPER (Capsicum chinense JACQ.)}

\begin{abstract}
The demand for the consumption of Biquinho pepper Capsicum chinense Jacq. is increasing and one of the problems in its production chain being the loss of mass and typical characteristics of the fruit. Thus, the study of alternatives on post-harvest conservation has great importance for producers, processors and consumers. In this way, the objective was to evaluate the use of edible toppings for the conservation of peppermint fruits. Washed and sanitized fruits were immersed in carnauba wax, cassava starch, gelatin and citrus pectin wrapping and stored at $25^{\circ} \mathrm{C}$ ambient temperature. Fruits washed and only sanitized constituted one of the treatments and fruits only washed were the control. At zero and at every 3 days for 12 days, physicalchemical, microbiological and visual evaluation of fruits were performed. There was no difference $(p>0.05)$ for $\mathrm{pH}$, titratable acidity and soluble solids content between treatments, however there was a difference $(p<0.05)$ in relation to storage time. For firmness there was no difference $(p>0.05)$ between treatments. The red or yellow coloration of the fruits remained stable during storage, regardless of the treatment. The Chroma index
\end{abstract}

\footnotetext{
${ }^{1,2}$ Cientistas de Alimentos, Instituto Federal de Educação, Ciência e Tecnologia do Sudeste de Minas Gerais, Rio Pomba, MG; natalyalmeida20@gmail.comegrazi.oliveira1993@hotmail.com;

${ }^{2}$ Pesq. Embrapa/Epamig Sudeste, Viçosa, MG; cleide@epamig.br;

${ }^{3,4}$ Pesq. Epamig Sudeste; claudia@epamig.br; e slopes@epamig.br;

${ }^{5}$ Prof. Instituto Federal de Educação, Ciência e Tecnologia do Sudeste de Minas Gerais, Rio Pomba, MG; eliane.martins@ifsudestemg.edu.br.
} 
varied $(p<0.05)$ with storage time. In all treatments mass loss was observed throughout the storage time. It was concluded that the physico-chemical characteristics such as $\mathrm{pH}$, titratable acidity, soluble solids content, firmness and staining are not altered with the use of edible toppings. However, these same coatings do not contribute to minimize the loss of fruit mass with consequent occurrence of wrinkling and browning at the end of 12 days of storage. The 3\% manioc starch coating allows to maintain the visual characteristics of black pepper for 12 days. The removal of the peduncle may have contributed to the increase of the microbial contamination of the fruits.

Keywords: biofilms, Capsicum, comestible coating, post-harvest, shelf life.

\section{INTRODUÇÃO}

A pimenta-biquinho, também conhecida pelo nome de pimenta-de-bico, sem pungência ou ardume, possui frutos aromáticos, crocantes, saborosos e atendem à demanda de consumidores que não apreciam pimentas com ardume. Associado às suas características sensoriais e nutricionais, a demanda de mercado desta pimenta é crescente sendo um dos entraves na cadeia produtiva a ocorrência de alterações pós-colheita como perda de massa e de características típicas do fruto.

Após a colheita, as pimentas são altamente perecíveis sendo afetadas por alguns processos degradativos relacionados com a temperatura e umidade relativa do ambiente de armazenamento ou de comercialização. Dentre os maiores problemas ocasionados por estes processos estão à rápida perda de água dos frutos após a colheita, que resulta em murchamento e a descoloração do pedúnculo, com perda da coloração verde característica. Estes dois problemas reduzem o valor do produto no mercado e podem ser motivos de descarte na comercialização. Além desses fatores, há danos sofridos pelo frio (pequenas manchas superficiais esbranquiçadas dispersas na superfície do pericarpo), quando armazenadas em temperaturas inferiores a $7^{\circ} \mathrm{C}$ (Marques et al. 2005). Como recomendação geral, as pimentas devem ser armazenadas entre 7 e $10{ }^{\circ} \mathrm{C}$ e com umidade relativa do ar entre 90 e $95 \%$. Porem esta condição de umidade do ar dificilmente é obtida na maioria das unidades comerciais de refrigeração ou refrigerador doméstico, visto que as unidades não dispõem de sistemas de controle de umidade. Neste caso, poderá ocorrer desidratação rápida dos frutos (Finger \& Vieira, 1997).

A busca de alternativas que propiciem o aumento da vida útil das pimentas no ambiente de comercialização à temperatura ambiente, constitui uma preocupação para preservar a qualidade dos frutos, minimizar perdas e prolongar a vida de prateleira (Chitarra et al., 1990; Pinheiro, 2012). Dentre as técnicas pós-colheita podese citar o uso de coberturas denominadas comestíveis ou biofilmes comestíveis ou ainda revestimentos comestíveis, bastante exploradas no revestimento de hortaliças frescas e frutas com o objetivo de melhorar ou substituir algumas características apresentadas pelas camadas da epiderme natural. Essas coberturas funcionam como barreira às trocas gasosas e à perda de água e protegem os produtos da degradação física, química e biológica resultando em extensão da vida de prateleira das hortaliças e frutas (Fakhouri et al., 2007; Morgado et al., 2008; Amariz et al., 2010; Castricini et al., 2012; Pinheiro, 2012; Oliveira \& Cereda, 2003 \& Oliveira; Santos, 2015). Esse aumento no interesse por coberturas comestíveis biodegradáveis (cera de carnaúba, fécula de mandioca, gelatina, pectina e outros) está pautado na demanda da sociedade por alimentos de qualidade, na preocupação ambiental com descarte de materiais não renováveis e na oportunidade para se criar novos mercados de matérias-primas biodegradáveis, de baixo custo, resistente, transparente e que atua como barreira à perda de água, tornando hortaliças e frutas comercialmente atrativas ao proporcionar mais brilho para a superfície e, não sendo tóxica, pode ser consumida. Os biofilmes comestíveis são inodoros, biodegradáveis e seguros do ponto de vista de segurança do alimento (Scanavaca et al., 2007; Akhtar et al., 2012; Al-Hassan \& Norziah, 2012; Dhall, 2013; Elsabee \& Abdou, 2013). Por serem aplicadas diretamente sobre a superfície dos frutos e fazerem parte do produto, os materiais empregados em sua elaboração devem ser considerados GRAS (Generally Recognized as safe), seguros para o uso em alimentos (Assis \& Britto, 2014; FDA, 2018).

Diante do exposto, objetivou-se avaliar o uso de coberturas comestíveis para fins de conservação póscolheita de pimenta-biquinho. 


\section{MATERIAL E MÉTODOS}

O trabalho foi desenvolvido nos Laboratórios de Microbiologia de Alimentos e de Análise de Alimentos (DCTA III) da Unidade de Processamento de Frutas e Hortaliças do Departamento de Ciência e Tecnologia de Alimentos do Instituto Federal de Educação, Ciência e Tecnologia do Sudeste de Minas Gerais, Campus Rio Pomba (IF Sudeste MG). Frutos sem pedúnculo, isentos de injúrias causadas por fungos/e ou insetos e de outras lesões, foram lavados em água corrente, sanitizados com solução clorada $150 \mathrm{mg}$. L $\mathrm{L}^{-1}$, por 10 min, drenados para retirada do excesso da solução sanitizante, e submetidos aos seguintes tratamentos: A0) frutos apenas lavados; A1; frutos apenas sanitizados; A2) frutos sanitizados imersos em cera de carnaúba pura; A3) frutos sanitizados imersos em cera de carnaúba diluída em água na proporção 1:1; A4) frutos sanitizados imersos em fécula de mandioca 3\%; A5) frutos sanitizados imersos em gelatina 3\%; A6) frutos sanitizados imersos em gelatina $1,5 \%$ + fécula de mandioca $1,5 \%$ e; A7) frutos sanitizados imersos em pectina cítrica de alta metoxilação $2,0 \%$. A fécula de mandioca, a gelatina e a pectina foram dissolvidas em água a, aproximadamente, $70{ }^{\circ} \mathrm{C}$ e, em seguida, mantidas em repouso para o resfriamento em temperatura ambiente. As pimentas foram imersas nas diversas coberturas por cerca de 30 segundos, drenadas, submetidas à secagem em secador elétrico (marca TAIFF, modelo SEC, turbo 6000), a $45^{\circ} \mathrm{C}$, por cinco minutos e, acondicionadas em bandejas plásticas mantidas a temperatura ambiente de $25^{\circ} \mathrm{C}$ e umidade relativa ambiente para a avaliação da vida de prateleira.

As coberturas foram avaliadas previamente quanto ao $\mathrm{pH}$ e acidez total titulável em \% de ácido cítrico das coberturas comestíveis.

\section{Análises físico-químicas}

No tempo zero e a cada três dias, durante 12 dias de armazenamento, foram realizadas análises físicoquímicas de $\mathrm{pH}$, acidez titulável (AT), teor de sólidos solúveis firmeza, cor superficial e índice croma. Ao longo do armazenamento, avaliou-se a perda de massa. Determinou-se o pH com peagâmetro Tecnopon modelo NT PHN, previamente calibrado (Zenebon et al., 2008). A acidez total titulável (Zenebon et al., 2008) foi expressa em porcentagem de ácido cítrico, que é o ácido orgânico presente em maior quantidade nos frutos de pimenta. A análise do teor de sólidos solúveis foi realizada em refratômetro de bancada tipo ABBE (Zenebon et al., 2008) sendo os resultados expressos em ${ }^{\circ}$ Brix. A firmeza dos frutos foi determinada com utilização de um penetrômetro digital da marca Impac, modelo FR-5120, com sonda cilíndrica de $3 \mathrm{~mm}$., em 3 frutos aleatórios por amostra, considerandose a média dos valores dos três frutos. Os resultados foram expressos em Newton (N).

Para a determinação da cor utilizou-se um colorímetro Konica Minolta modelo CR-10 e leitura direta de reflectância das coordenadas $\mathrm{L}^{*}, \mathrm{a}^{*} \mathrm{e} \mathrm{b}$, empregandose a escala Hunter-Lab. A coordenada L* (luminosidade) varia de 0 para o preto, até 100 para o branco, a* (valor positivo, uma medida do vermelho e negativo, uma medida do azul). A partir dos valores de $\mathrm{L}^{*}, \mathrm{a}^{*} \mathrm{e} \mathrm{b}^{*}$ foram calculados o índice colorimétrico Chroma $\left(\mathrm{C}^{*}\right)$ ou índice Chroma, que define uma medida de saturação ou intensidade de cor e varia de cores pálidas (valor baixo) para cores vivas (valor alto) e é calculado pela equação: $C^{*}=\left(a^{2}+b^{2}\right)^{1 / 2}$ (Oliveira et al., 2010).

A perda de massa foi determinada a partir de 10 frutos pesados em balança analítica digital da marca Marte modelo AY220 com precisão de $0,01 \mathrm{~g}$. Os resultados foram expressos em porcentagem, considerando-se a diferença entre a massa inicial e aquela obtida a cada 3 dias de armazenamento, por meio da seguinte equação:

$\operatorname{PM}(\%, \mathrm{~m} / \mathrm{m})=[($ massa inicial - massa final $)] \times 100$ [(massa inicial)]

A avaliação visual das pimentas foi realizada no tempo zero e a cada três dias, atribuindo-se as seguintes notas e classificações/descrições: 1 - Péssimo (fruto murcho, com presença de fungos e escurecido); 2 Ruim (fruto murcho e com fungos); 3 - Regular (fruto murcho e sem fungos); 4 - Bom (fruto sem fungos e turgido); 5 - Ótimo (fruto túrgido, sem fungos e cor normal) (Korte \& Favarão, 2016).

\section{Análises microbiológicas}

As análises de coliformes termotolerantes, a $45^{\circ} \mathrm{C}$ foram realizadas pela técnica do número mais provável (Kornacki \& Johnson, 2001) no início do armazenamento (tempo zero) e no final da vida útil dos frutos (Brasil, 2001). Para a enumeração de fungos filamentosos e leveduras, alíquotas de $25 \mathrm{~g}$ de frutos, foram diluídas em $225 \mathrm{~mL}$ de solução salina peptonada $(0,85 \% \mathrm{NaCl}$ e $0,1 \%$ peptona $)$, seguindo-se diluições seriadas. Foi utilizado o método de espalhamento em 
superfície (Spread Plate) com adição de 0,1 mL da diluição da amostra na superfície do meio de cultura Agar Batata Dextrose (BDA) acidificado com ácido tartárico 10\%. As placas foram incubadas a $25^{\circ} \mathrm{C}$, por cinco dias, em estufa BOD Novatecnica modelo NT 704. Consideraramse para contagens, placas com 15 a 150 colônias. Os resultados foram expressos em UFC/g de amostra.

\section{Análises estatísticas}

Foi utilizado o delineamento inteiramente casualizado com três repetições em duplicata. Os dados foram submetidos à análise de variância fatorial (teste $F$ ) e, as médias, comparadas por meio do Teste de Tukey (5\%) utilizando-se do programa Dell Inc. (2015) Dell Statistica, version 13 .

\section{RESULTADOS E DISCUSSÃO}

Os valores de pH e de acidez titulável (AT), em percentagem de ácido cítrico, das coberturas antes da imersão dos frutos são apresentados na Tabela 1 .

\section{pH, acidez titulável, sólidos solúveis}

Não houve interação significativa entre tratamentos e tempo de armazenamento para $\mathrm{pH}$, acidez e sólidos solúveis. Entre tratamentos também não houve diferença para estas três características $(p>0,05)$. Embora os valores de $\mathrm{pH}$ das coberturas, antes da imersão dos frutos, tenham variado de 3,62 para a pectina cítrica a 6,97 para a cera de carnaúba pura (Tabela 1), os tratamentos não diferiram entre si após a imersão nas diferentes coberturas.

Os valores de $\mathrm{pH}$, acidez e sólidos solúveis foram influenciados $(\mathrm{p}<0,05)$ pelo tempo de armazenamento (Tabela 2). Para o pH, a diferença foi constatada apenas entre os valores no tempo zero e 12 dias de armazenamento.

Tabela 1 - Valores de pH e acidez das coberturas comestíveis utilizadas para tratamento de frutos de pimenta-biquinho

\begin{tabular}{lcc}
\hline Coberturas & $\mathrm{pH}$ & Acidez \\
\hline Cera de carnaúba pura & 9,77 & $\mathrm{ND}$ \\
Cera de carnaúba diluída em água $(1: 1)$ & 9,64 & $\mathrm{ND}$ \\
Fécula de mandioca 3\% & 6,97 & 0,006 \\
Gelatina diluída 3\% & 6,09 & $\mathrm{ND}$ \\
Fécula de mandioca 1,5\%+ gelatina 1,5\% & 6,31 & 0,018 \\
Pectina cítrica 2\% & 3,62 & 0,15 \\
\hline
\end{tabular}

ND: não determinado.
Tabela 2 - Média dos valores de $\mathrm{pH}$, acidez e ${ }^{0}$ Brix de pimenta-biquinho referentes aos tratamentos durante 12 dias de estocagem à temperatura ambiente de $25^{\circ} \mathrm{C}$

\begin{tabular}{clll}
\hline Tempo (dias) & pH & Acidez & ${ }^{0}$ Brix \\
\hline 0 & $5,18 \mathrm{a}$ & $0,22 \mathrm{ab}$ & $6,79 \mathrm{ab}$ \\
3 & $5,28 \mathrm{ab}$ & $0,25 \mathrm{~b}$ & $6,45 \mathrm{a}$ \\
6 & $5,45 \mathrm{ab}$ & $0,19 \mathrm{a}$ & $8,90 \mathrm{bc}$ \\
9 & $5,47 \mathrm{ab}$ & $0,19 \mathrm{a}$ & $7,85 \mathrm{abc}$ \\
12 & $5,64 \mathrm{~b}$ & $0,21 \mathrm{ab}$ & $9,91 \mathrm{c}$ \\
\hline
\end{tabular}

Médias seguidas de mesma letra na coluna não diferem estatisticamente pelo teste de Tukey a $5 \%$ de probabilidade.

Para a acidez, não há como explicar a diferença entre 3 e 6 e entre 3 e 9 dias de armazenamento. Os valores de $\mathrm{pH}(5,18$ a 5,64$)$ observados foram próximos daqueles constatados em diversas espécies de pimenta $(4,98$ a 5,45) (Valverde, 2011; Borges et al., 2015). Segundo Chitarra \& Chitarra (2005), após a colheita e durante o armazenamento, as concentrações dos ácidos orgânicos diminuem em decorrência de sua utilização como substrato, na respiração, ou da sua transformação em açúcares. Porém, em alguns casos, há um pequeno aumento nos valores com o avanço da maturação. Do ponto de vista comercial, é desejável que os frutos apresentem menor $\mathrm{pH}$ e maior acidez o que representa menores riscos de deterioração e contaminação por microrganismos prejudiciais à saúde humana. As células microbianas são substancialmente afetadas pelo $\mathrm{pH}$ dos alimentos sendo que cada microrganismo tem um pH ótimo de crescimento (Engelkirk, 2012).

O teor de sólidos solúveis foi mais elevado no tempo de 12 dias comparado àquele observado nos tempos zero, 3 e 6 dias de armazenamento. Os sólidos solúveis, designados como ${ }^{0} \mathrm{BRIX}$, indicam a quantidade de sólidos que se encontram dissolvidos no suco ou na polpa das frutas e, apresentam tendência de aumento com a maturação, seja por biossíntese, pela degradação de polissacarídeos, ou, ainda, pela excessiva perda de água dos frutos (Chitarra \& Chitarra, 2005). A variação dos sólidos solúveis durante o amadurecimento e armazenamento é composta em grande parte por açucares que compõem o sabor dos frutos, em equilíbrio com os ácidos orgânicos. Quando ocorre perda de massa há incremento no teor de sólidos solúveis, associado a concentração dos teores de açúcares no interior dos tecidos (Kluge \& Minami, 1997; Chaves \& Furtado, 2015). É importante ressaltar que os sólidos solúveis, 
provavelmente, contribuíram para deterioração dos frutos sem pedúnculo, ocasionada pela ação de microrganismos que necessitam dos açúcares como fonte de energia para crescimento e desenvolvimento.

\section{Firmeza}

Não houve interação significativa entre tratamentos e o tempo de armazenamento para firmeza de frutos. Não houve diferença de firmeza $(\mathrm{p}>0,05)$ entre os frutos dos diferentes tratamentos embora aqueles imersos em gelatina $1,5 \%$ associada a fécula de mandioca $1,5 \%$ (A6) tenham se apresentado mais firmes. Não houve também diferença de firmeza ( $p>0,05)$ dos frutos com o tempo de armazenamento.

Após a colheita, a perda excessiva de água pela transpiração pode comprometer consideravelmente a firmeza das frutas, fazendo com que essas se apresentem flácidas, moles e murchas (Chitarra \& Chitarra, 2005). O penetrômetro é o aparelho mais usado para determinar a firmeza de hortaliças e frutas, porém, não é muito útil para avaliar a variação de firmeza causada por desidratação a exemplo do ocorrido com frutos de pimentabiquinho. Isso decorre do fato de que, em geral, a desidratação não é acompanhada do enfraquecimento da cimentação entre as células na lamela média. Em vista disso, por aumento de elasticidade é comum ocorrer um aumento do valor da leitura da força de penetração em produtos submetidos à desidratação. A desidratação, por se tratar de uma componente de firmeza que depende da turgescência celular, é preferível empregar um instrumento mais apropriado como o aplanador de turgescência desenvolvido na Embrapa Hortaliças (Calbo \& Carmelo, 2017).

Uma oscilação no teor de firmeza foi observada ao utilizar filmes comestíveis na conservação pós-colheita de pimentões 'Magali R' (Lemos, 2007). A maior perda de massa, provavelmente, promoveu uma maior resistência à penetração da ponteira do penetrômetro na superfície do fruto de pimentão (Lemos, 2007), em pitaias (Brunini \& Cardoso, 2011) e em mangas (Jeronimo et al. (2007). Esses autores atribuíram essa maior firmeza à perda de massa fresca $\mathrm{e}$, assim, $\mathrm{o}$ murchamento $\mathrm{e}$ flacidez dos vegetais.

\section{Análise de cor}

A colorimetria tem sido utilizada para caracterizar a cor de diferentes pigmentos a exemplo das antocianinas, clorofila e carotenoides, bem como para avaliar a qualidade de um produto "in natura" ou processado, sendo um fator determinante da vida útil de um produto (Lima et al., 2007).

Em todos os tratamentos, os frutos apresentaram baixos valores de $\mathrm{L}^{*}$ indicativos de baixa luminosidade. Da mesma forma, Mattiuz et al. (2009) observaram em uvas armazenadas à temperatura ambiente menor valor médio de $\mathrm{L}$ comparadas àquelas armazenadas a $12{ }^{\circ} \mathrm{C}$, com aspecto escurecido. Ao contrário, Cerqueira (2012) observou por meio do aumento dos valores de $L^{*}$, a perda de cor ou clareamento de pimentas embaladas em frascos de polipropileno.

Os valores das coordenadas $a^{*}$ e b* foram permanentemente positivos (Tabela 3 ) demonstrativo da tendência a coloração vermelha e amarela as quais se mantiveram estáveis durante o armazenamento.

A diferença não significativa $(p>0,05)$ entre tratamentos para o índice Chroma (Tabela 3), indicou que as coberturas mantiveram a coloração viva do fruto semelhante aos frutos dos tratamentos (sem cobertura) e sem influenciar no seu brilho, fator importante, pois a cor é um dos principais atributos de qualidade observados pelos consumidores na hora da compra (Chitarra \& Chitarra, 2005). Alto valor de índice Chroma de é indicativo de cores vivas (Goyenèche et al., 2014).

Houve influência $(p<0,05)$ do tempo de armazenamento sobre o índice Chroma ( $\mathrm{C}^{*}$ ) (Tabela 4) com aumento nos valores entre os tempos 3 e 6 dias, período em que se observou uma maior intensidade de cor dos frutos. A partir de 9 dias, houve decréscimo no valor de $\left(\mathrm{C}^{*}\right)$ alteração esta indicativa de redução

Tabela 3 - Cor dos frutos de pimenta-biquinho nos diferentes tratamentos durante o armazenamento a temperatura ambiente de $25^{\circ} \mathrm{C}$

\begin{tabular}{cccc}
\hline Tratamentos & $\mathrm{L}^{*}$ & $\mathrm{a}^{*}$ & $\mathrm{~b}^{*}$ \\
\hline A0 & 17,98 & $+30,54$ & $+15,50$ \\
A1 & 21,91 & $+34,83$ & $+16,76$ \\
A2 & 21,98 & $+32,05$ & $+15,95$ \\
A3 & 21,97 & $+33,34$ & $+16,46$ \\
A4 & 21,39 & $+34,16$ & $+17,17$ \\
A5 & 22,56 & $+34,88$ & $+15,78$ \\
A6 & 23,10 & $+34,74$ & $+17,21$ \\
A7 & 20,12 & $+34,45$ & $+16,75$ \\
\hline
\end{tabular}


Tabela 4 - Média dos valores do índice Chroma dos frutos de pimenta-biquinho referentes aos tratamentos ao longo do armazenamento à temperatura ambiente de $25^{\circ} \mathrm{C}$

\begin{tabular}{cl}
\hline Tempo ( dias) & \multicolumn{1}{c}{ Cor } \\
\hline 0 & $21,8 \mathrm{c}$ \\
3 & $23,1 \mathrm{bc}$ \\
6 & $26,1 \mathrm{a}$ \\
9 & $25,2 \mathrm{ab}$ \\
12 & $22,9 \mathrm{bc}$ \\
\hline
\end{tabular}

Médias seguidas de mesma letra na coluna não diferem estatisticamente pelo teste de Tukey a $5 \%$ de probabilidade.

da intensidade da coloração associada a desidratação parcial da superfície dos frutos. A aplicação de cobertura comestível em cenoura resultou em decréscimos do $\mathrm{C}^{*}$ ao longo do tempo, fato atribuído a perda de água da superfície da hortaliça (Fai et al., 2015). Observouse, em cajus revestidos com cera de carnaúba e triclosan, maiores valores de $\mathrm{C}^{*}$ quando comparados aos revestidos com ácido sórbico e àqueles sem revestimento (Pinheiro, 2012).

Dentre as características sensoriais, a cor tem papel fundamental na avaliação da qualidade de pimenta. Em geral, a cor é utilizada como indicador de qualidade e maturação dos frutos e, consequentemente, do aroma, textura, valor nutritivo e mesmo a integridade do vegetal (Fernandes \& Souza, 2001).

\section{Perda de massa e avaliação visual dos frutos}

Não houve interação significativa entre tratamentos e o tempo de armazenamento, para perda de massa. Constatou-se variação na perda de massa dos frutos de $11,5 \%$ a $13,7 \%$ sendo que não houve diferença $(p>0,05)$ entre os tratamentos, mas houve perda de massa $(p<0,05)$ nos frutos com aumento de perda ao longo dos 12 dias de avaliação (Figura 1), resultados associados à perda de água com consequente desidratação dos frutos (Chitarra \& Chitarra, 2005).

A perda de água pode ser tolerada, mas aquela responsável pelo murchamento ou enrugamento deve ser evitada. Perdas de 3\% a $6 \%$ foram suficientes para causar um declínio marcante de qualidade, entretanto, alguns produtos são ainda comercializáveis com $10 \%$ de perda de umidade ou mesmo $15 \%$ no caso de pimentão (Bussel \& Kenisgberger, 1975). A perda de massa fresca, associada à perda de água, que ocorre no armazenamento

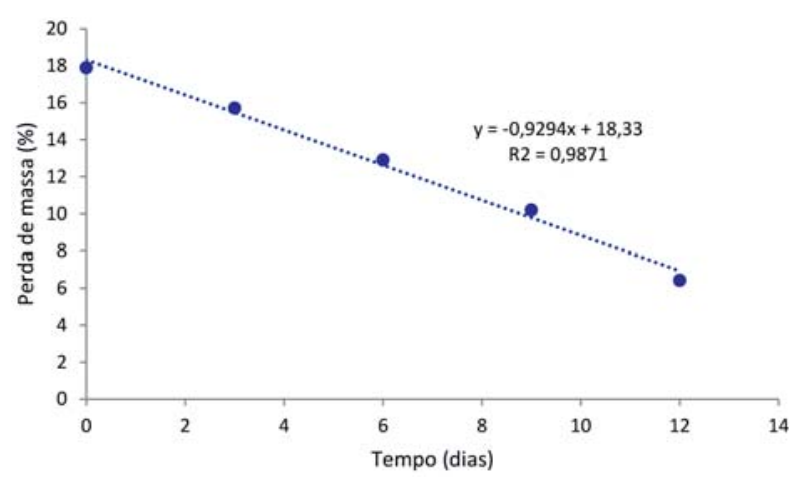

Figura 1 - Valores de perda de massa (\%) de frutos de pimenta-biquinho referentes aos tratamentos, durante 12 dias de armazenamento à temperatura ambiente de $25^{\circ} \mathrm{C}$.

dos frutos implica em desvalorização comercial, uma vez que os frutos apresentam não apenas alterações quantitativas mas, também, na aparência incluindo a cor e murchamento da casca, firmeza e perda de frescor, qualidade nutricional do produto, embora a polpa, mesmo assim, possa apresentar boas condições de consumo (Kader, 2002; Lemos, 2007; Brunini \& Cardoso, 2011).

A perda de massa constatada em frutos de pimenta dedo-de-moça revestidos com cera de carnaúba foi $26 \%$ inferior comparado ao tratamento controle o que refletiu no aspecto dos frutos durante o armazenamento (Pinto et al., 2009). Esses autores concluíram que o uso de revestimentos com cera de carnaúba para a conservação pós-colheita de frutos in natura de pimenta Dedo-de-moça, nas condições avaliadas, constitui uma alternativa para o aumento da vida de prateleira do produto.

Filmes derivados de fécula da mandioca, quando aplicados em frutos de pimentão armazenados, por 10 dias, em condição ambiente, não foram eficientes como barreira à perda de água (Vicentini et al., 1999). Segundo Lima et al. (2014), melhores resultados com a utilização de coberturas comestíveis, são obtidos quando estas são combinadas com refrigeração. A aplicação de película de fécula de mandioca na concentração mais elevada (4\%) e sob refrigeração, proporcionou ao pepino um aspecto melhor de conservação, tornando o produto mais atraente (Reis et al., 2006). A proteção de frutos de pimentão com cera de carnaúba foi favorável à manutenção da massa fresca sem prejuízos aos teores de sólidos solúveis, acidez titulável e ácido ascórbico, 
com aumento significativo na vida útil para até 33 dias com o armazenamento a $5^{\circ} \mathrm{C}$ (Morgado et al., 2008).

Desta forma, pode-se sugerir que a impossibilidade de seleção de uma melhor cobertura para conservação de pimenta-biquinho no presente estudo para fins de minimizar a perda de massa dos frutos durante o armazenamento pode ser atribuída ao armazenamento em temperatura ambiente. Assim, é recomendável o efeito de coberturas comestíveis em frutos armazenados à baixas temperaturas.

\section{Aparência visual}

Constatou-se, nos frutos, a presença de pontos escuros na superfície, de fungos filamentosos, murchamento, amolecimento e deterioração. Observouse também que algumas coberturas contribuíram para a conservação do brilho por maior de tempo. Com 3 dias de armazenamento, nos tratamentos sem cobertura assim como naqueles revestidos com cera de carnaúba pura ou diluída e com pectina cítrica constatou-se maior quantidade de frutos com sinais de murchamento na região do pedúnculo e enrugamento na superfície externa. Já nos demais tratamentos (frutos imersos em fécula de mandioca $3 \%$, em gelatina diluída $3 \%$ e em fécula de mandioca a $1,5 \%$ combinada com gelatina a $1,5 \%$ ) os frutos se apresentaram mais conservados, com maior brilho e com poucas alterações na superfície próxima ao pedúnculo (Figura 2).

Com 12 dias de armazenamento dos frutos sem cobertura assim como aqueles imersos em cera de carnaúba pura e diluída, observou-se grande parte da superfície escurecida e enrugada, contaminação fúngica na região do pedúnculo, inadequados para o consumo e comercialização. Já os frutos imersos em fécula de mandioca $3 \%$, gelatina diluída $3 \%$, fécula de mandioca $1,5 \%$ + gelatina $1,5 \%$ e em pectina cítrica, apresentaramse murchos, com superfície escurecida, porém com baixo crescimento de fungos na região do pedúnculo, mas, em sua maioria, em condições inadequadas para consumo e comercialização (Figura 3). Dentre estes três tratamentos, frutos imersos em fécula de mandioca a $3 \%$ apresentaram melhor aspecto visual com relação à cor e brilho, ao qual foi atribuída a nota 3 , nota correspondente a frutos de aparência regular, aos 12 dias de armazenamento (Figura 4). Bolzan (2008) também observou que o uso de biofilme à base de fécula de mandioca para conservação de tomates conferiu ao produto melhor aparência, coloração e firmeza de polpa.

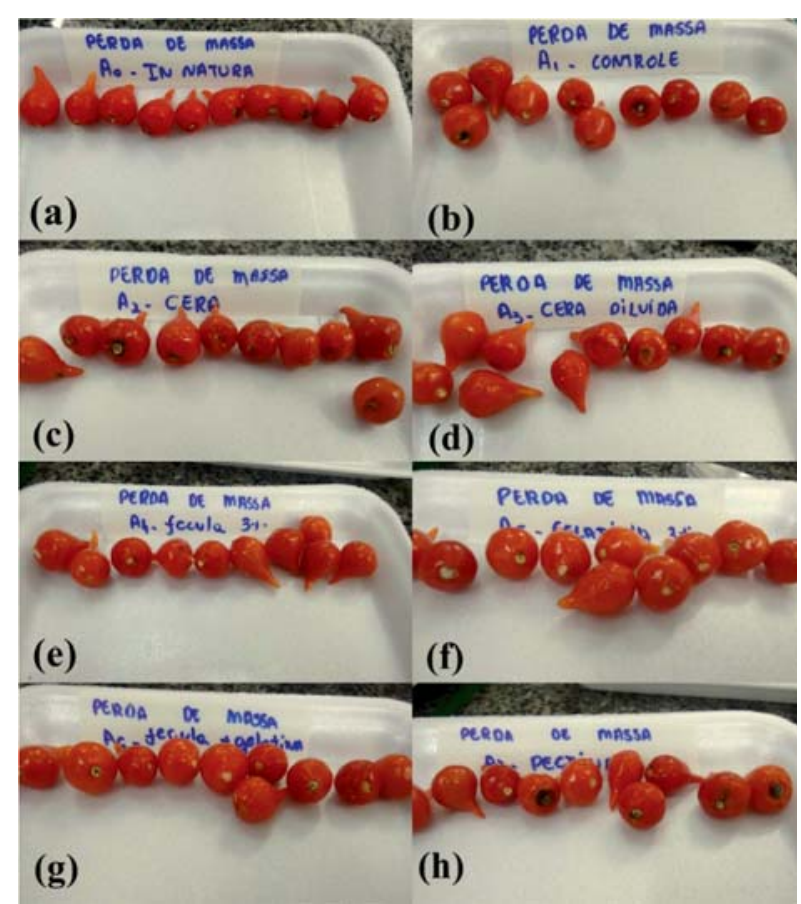

Figura 2 - Pimenta-biquinho com três dias de armazenamento à temperatura ambiente de $25^{\circ} \mathrm{C}$. (a) frutos não sanitizados; (b) frutos sanitizados; (c) frutos imersos em cera de carnaúba; (d) frutos imersos em cera de carnaúba diluída em água 1:1; (e) frutos imersos em fécula de mandioca $3 \%$; (f) frutos imersos em gelatina $3 \%$; (g) frutos imersos em fécula de mandioca $1,5 \%$ + gelatina $1,5 \%$ e (h) frutos imersos em pectina cítrica $2 \%$.

\section{Determinação das características microbiológicas}

As contagens foram acima dos parâmetros estabelecidos pela legislação vigente para a primeira e segunda repetições (Tabela 5). De acordo com a RDC n. ${ }^{\circ} 12$, os padrões de seguridade microbiológica para frutas frescas in natura preparadas, é de no máximo, $5 \times 10^{2}$ UFC. $\mathrm{mL}^{-1}$ de coliformes a $45^{\circ} \mathrm{C}$ por grama do produto (Brasil, 2001).

A baixa qualidade microbiológica dos frutos adquiridos para realização da primeira e segunda repetições associadas à retirada previa do pedúnculo dos frutos pelos produtores pode ter propiciado a contaminações nos frutos. Já os frutos usados na terceira repetição apresentaram padrões microbiológicos de acordo com a RDC n. ${ }^{\circ} 12$ (Brasil, 2001). Esse resultado pode ser atribuído à boa qualidade microbiológica dos 
frutos os quais foram adquiridos com pedúnculo, situação que previne a contaminação dos frutos. A retirada do pedúnculo foi feita realizada antes da sanitização

Frutos de pimenta referentes aos tratamentos apresentaram resultados insatisfatórios para fungos filamentosos e leveduras (Tabela 6) observados por meio das altas contagens no início do armazenamento (zero dia) e no final, com 12 dias. A retirada do pedúnculo e a exposição dos frutos à temperatura ambiente no armazenamento são fatores que favorecem o crescimento desses microrganismos. Jung e Degenhardt (2016) observaram, em tomate cereja tratado com cobertura bioativa, que a presença do pedúnculo é importante por constituir uma barreira ao acesso de microrganismos, uma vez que previne o surgimento de fissuras. O baixo $\mathrm{pH}$ proporcionado

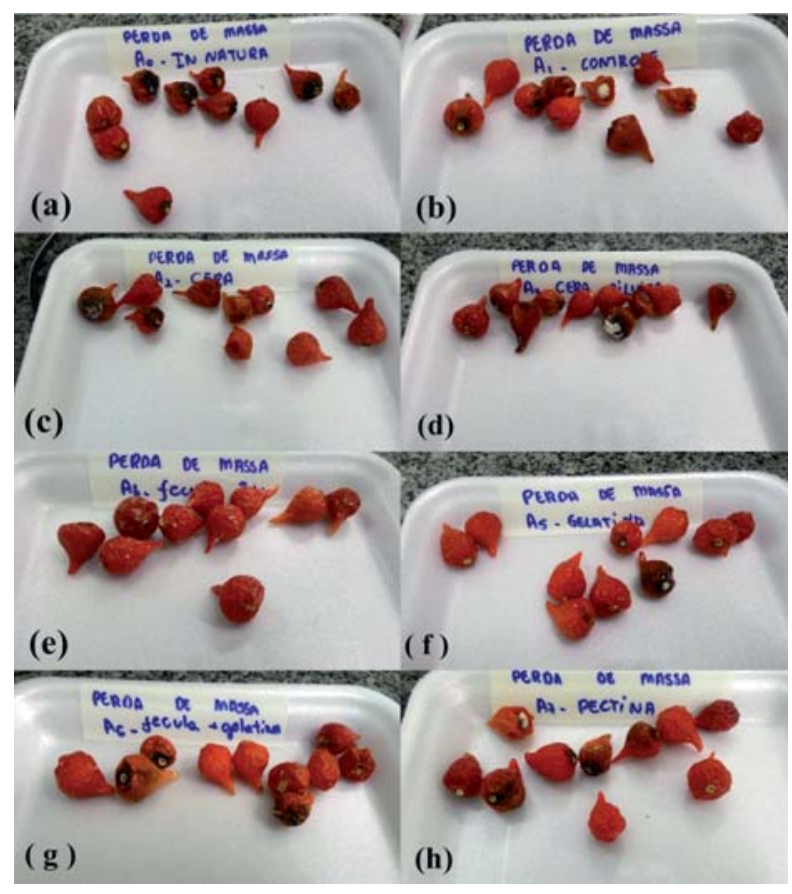

Figura 3 - Pimenta-biquinho com 12 dias de armazenamento a temperatura ambiente. (a) frutos não sanitizados; (b) frutos sanitizados; (c) frutos imersos em cera de carnaúba; (d) frutos imersos em cera de carnaúba diluída em água $1: 1$; (e) frutos imersos em fécula de mandioca $3 \%$; (f) frutos imersos em gelatina $3 \%$; $(\mathrm{g})$ frutos imersos em fécula de mandioca $1,5 \%+$ gelatina $1,5 \%$ e (h) frutos imersos em pectina cítrica $2 \%$.

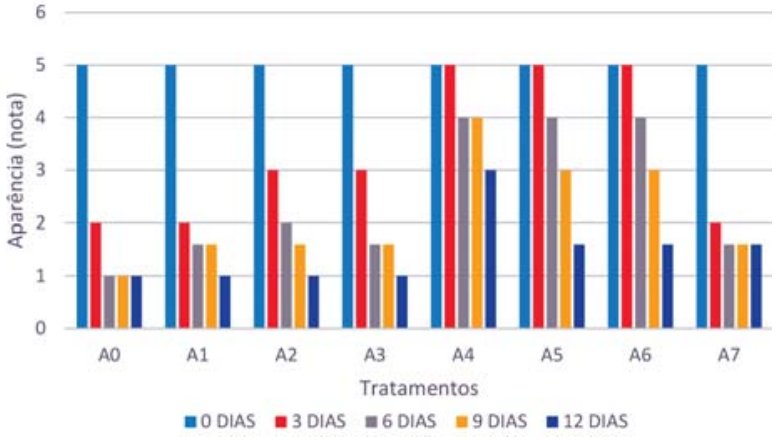

Figura 4 - Aparência dos frutos de pimenta-biquinho submetidos aos diferentes tratamentos. A0 - frutos não sanitizados; A1- frutos sanitizados; A2 frutos imersos em cera de carnaúba; A3 - frutos imersos em cera de carnaúba diluída em água 1:1; A4 - frutos imersos em fécula de mandioca 3\%; A5 frutos imersos em gelatina 3\%; A6 - frutos imersos em fécula de mandioca $5 \%$ + gelatina $1,5 \%$ e A 7 - frutos imersos em pectina cítrica $2 \%$.

Notas de aparência: 1- Péssimo (fruto murcho, com fungos e escurecido); 2- Ruim (fruto murcho e com fungos); 3- Regular (fruto murcho e sem fungos); 4-Bom (fruto sem fungos e túrgido); 5- Ótimo (fruto túrgido, sem fungos e cor normal).

pela pectina cítrica $2 \%$ pode ter limitado o crescimento de fungos filamentosos e de leveduras, com redução da contaminação fúngica.

\section{CONCLUSÕES}

Os revestimentos cera de carnaúba pura ou diluída, fécula de mandioca a 1,5\% combinado com gelatina a $1,5 \%$, gelatina a $3 \%$ e pectina cítrica a $2 \%$ não influenciam nas características físico-químicas de $\mathrm{pH}$, acidez titulável, teor de sólidos solúveis, firmeza e coloração da pimenta-biquinho. Entretanto, estes não contribuem para prevenir a perda de massa de pimentabiquinho considerando que os frutos apresentaram, ao final do armazenamento, aspecto enrugado e escurecido. A perda de massa associada a perda de água durante o armazenamento é um fator limitante para a sua conservação pós-colheita e está diretamente relacionada com a aceitação de mercado.

A cobertura à base de fécula de mandioca a $3 \%$ permite a manutenção da qualidade visual da pimentabiquinho por 12 dias de armazenamento. 
Tabela 5 - Análises microbiológicas em pimenta-biquinho

\begin{tabular}{ccccc}
\hline \multirow{2}{*}{ Repetições } & \multicolumn{2}{c}{ Coliformes termotolerantes } & \multicolumn{2}{c}{ Escherichia coli } \\
\cline { 2 - 5 } & Zero dia & $12 \mathrm{dias}$ & Zero dia & $12 \mathrm{dias}$ \\
Primeira & $1,4 \times 10^{5} \mathrm{NMP} / \mathrm{g}$ & $2,4 \times 10^{4} \mathrm{NMP} / \mathrm{g}$ & $<3 \mathrm{NMP} / \mathrm{g}$ & $<3 \mathrm{NMP} / \mathrm{g}$ \\
Segunda & $7,7 \times 10^{5} \mathrm{NMP} / \mathrm{g}$ & $1,6 \times 10^{5} \mathrm{NMP} / \mathrm{g}$ & $17,6 \mathrm{NMP} / \mathrm{g}$ & $<3 \mathrm{NMP} / \mathrm{g}$ \\
Terceira & $<3,0 \mathrm{NMP} / \mathrm{g}$ & $6,7 \mathrm{NMP} / \mathrm{g}$ & $<3 \mathrm{NMP} / \mathrm{g}$ & $3,6 \mathrm{NMP} / \mathrm{g}$ \\
\hline
\end{tabular}

Tabela 6 - Média da contagem de fungos filamentosos e leveduras (UFC. g $^{-1}$ estimado) de frutos de pimenta-biquinho submetidos a diferentes tratamentos, em três repetições

\begin{tabular}{ccc}
\hline Tratamento & Zero dia & 12 dias \\
\hline A0 & $7,7 \times 10^{4}$ & $5,6 \times 10^{6}$ \\
A1 & $5,01 \times 10^{5}$ & $5,1 \times 10^{6}$ \\
A2 & $5,5 \times 10^{6}$ & $1,2 \times 10^{3}$ \\
A3 & $5,0 \times 10^{9}$ & $4,2 \times 10^{9}$ \\
A4 & $1,7 \times 10^{6}$ & $1,4 \times 10^{6}$ \\
A5 & $5,0 \times 10^{5}$ & $1,0 \times 10^{6}$ \\
A6 & $3,4 \times 10^{6}$ & $7,5 \times 10^{5}$ \\
A7 & $2,5 \times 10^{9}$ & $6,8 \times 10^{5}$ \\
\hline
\end{tabular}

A retirada do pedúnculo deve ser evitada considerando que contribui para o aumento da contaminação microbiana do fruto pós-colheita.

\section{AGRADECIMENTOS}

À Fundação de Amparo à Pesquisa do Estado de Minas Gerais - FAPEMIG pelo apoio financeiro à pesquisa.

\section{REFERÊNCIAS}

AKHTAR, M.J.; JACQUOT, M.; JASNIEWSKI, J.; JACQUOT, C.; IMRAN, M.; JAMSHIDIAN, M.; PARIS C.; DESOBRY, S. Antioxidant capacity and light-aging study of HPMC films functionalized with natural plant extract. Carbohydrate Polymers, v.89, p.1150-1158, 2012.

AL-HASSAN, A.A.; NORZIAH, M.H. Starchgelatin edible films: Water vapor permeability and mechanical properties as affected by plasticizers.

Food Hydrocolloids, v.6, p.108-11, 2012.

AMARIZ, A.; LIMA, M.A. C.; TRINDADE, D.C.G.; SANTOS, A.C.N.; RIBEIRO, T.P.

Recobrimentos à base de carboximetilcelulose $\mathrm{e}$ dextrina em mangas 'Tommy Atkins' armazenada sob refrigeração. Ciência Rural, v.40, p.2199$2205,2010$.

ASSIS, O.B.G.; BRITTO, D. Coberturas comestíveis protetoras em frutas: fundamentos e aplicações. Brazilian Journal of Food Technology, v.17, p.87-97, 2014.

BOLZAN, R.P. Biofilmes comestíveis para conservação pós colheita de tomate dominador. 2008. 152 f. Dissertação (Mestrado), Universidade Federal do Paraná, Curitiba, 2008.

BORGES, K.M.; VILARINHO, L.B.O.; FILHO, A.A.M.; MORAIS, B.S.; RODRIGUES, R.N.S. Caracterização morfoagronômica e físico-química de pimentas em Roraima. Revista Agro@ambiente On-line, v.9, p.292-299, 2015.

BRASIL. Agência Nacional de Vigilância Sanitária. Resolução RDC n. 12, de 02 de janeiro de 2001. Regulamento Técnico sobre padrões microbiológicos para alimentos. Diário Oficial da União, Brasília, DF, 10 de janeiro de 2001 .

BRUNINI, M.A.; CARDOSO, S.S. Qualidade de pitaias de polpa branca armazenadas e diferentes temperaturas. Revista Caatinga, v.24, n.3, p.78-84, 2011.

BUSSEL, J.; KENIGSBERGER, Z. Packaging green bell peppers in selected permeability films. Journal of Food Science, v.40, n.6, p.1300-1303, 1975.

CALBO, A.G.; CARMELO, G.P. Fisiologia póscolheita - métodos macroscópicos e instrumentos. In: FERREIRA, M.D (Ed.). Instrumentação póscolheita em frutas e hortaliças. Brasília, DF: Embrapa, 2017. 284 p. Disponível em: file://C:/ Users/User/Downloads/Embrapa-Instrumentacaoposcolheita.pdf. Acesso em: nov.2018. 
CASTRICINI, A.; CONEGLIAN, R.C.C.; DELIZA, R. Starch edible coating of papaya: effect on sensory characteristics. Ciência e Tecnologia de Alimentos, v.32, p.84-92, 2012.

CERQUEIRA, A.P. Conservação pós-colheita de pimentas-de-cheiro (Capsicum chinense) armazenadas sob atmosfera modificada e refrigeração. 2012. 66f. Dissertação (Mestrado) em Produção Vegetal, Universidade Federal do Tocantins, Gurupi Tocantins (TO). 2012.

CHAVES, A.A.C.; FURTADO, S.C. Análise físicoquímica da pimenta de cheiro mantida em temperatura ambiente. Revista Cientifica da Fametro, v.1, p.63-76, 2015.

ChitarRa, M.I.F.; ChitARRA, A.D. Póscolheita de frutos e hortaliças: fisiologia e manuseio. Lavras: ESAL/FAEPE, 1990. 320 p.

ChitarRa, M.I.F.; ChitarRA, A.B. Póscolheita de frutos e hortaliças: fisiologia e manuseio. 2. ed. Lavras: ESAL/FAEPE, 2005. 785 p.

DHALL, R.K. Advances in Edible Coatings for Fresh Fruits and Vegetables: A Review. Critical Reviews in Food Science and Nutrition, v.53, p.435-450, 2013.

ENGELKIRK, P.G; ENGELKIRK, J.D. Microbiologia para as ciências da saúde, 2012. 480p.

ELSABEE, M.Z.; ABDOU, E.S. Chitosan based edible films and coatings: A review. Materials Science and Engineering, v.33, p.1819-1841, 2013.

FAI, A.E.C.; SOUZA, M.R.A.; BRUNO, N.V.; GONÇALVES, E.C.B.A. Produção de revestimento comestível à base de resíduo de frutas e hortaliças: aplicação em cenoura (Daucus carota L.) minimamente processada. Scientia

Agropecuaria, v.6, p.59-68, 2015.

FAKHOURI, F.M.; FONTES, L. C. B.; GONÇALVES, P. V. M.; MILANEZ, C. R.; COLLARES-QUEIROZ, F. P. Filmes e coberturas comestíveis compostas à base de amidos nativos e gelatina na conservação e aceitação sensorial de uvas Crimson. Ciência e Tecnologia de Alimentos, Campinas, v.27, p.369-375, 2007.
FERNANDES, P.H.S.; SOUZA, S.D.O. Tecnologia de produtos de origem vegetal: processamento de frutas e hortaliças. Uberlândia, 2001. p.89-99.

FINGER, L.F.; VIEIRA, G. Controle da perda póscolheita de água em produtos hortícolas. Cadernos Didáticos 19. Viçosa: Editora UFV. 1997. 29p.

FOOD AND DRUG ADMINISTRATION - FDA Generally recognized as safe (GRAS). Silver Spring. Disponível em: <https://www.fda.gov/ food/ingredientspackaginglabeling/gras/> Acesso em: nov. 2018.

GOYENECHE, R.; AGÜERO, M.V.; ROURA, S.; SCALA, K.D. Application of citric acid and mild heat shock to minimally processed sliced radish: Color evaluation. Postharvest Biology and Technology, v.93, p. 106-113, 2014.

JERONIMO, E. M.; BRUNINI, M.A.; ARRUDA, M.C.; CRUZ, J.C.S.; FISCHER, I.H.; GAVA, G.J.C. Conservação pós-colheita de mangas 'Tommy Atkins' armazenadas sob atmosfera modificada. Semina: Ciências Agrárias, v.28, n.3, p.417-426, 2007.

JUNG, G.; DEGENHARDT, R. Polímero de recobrimento bioativo à base de amido de milho para prolongamento da vida de prateleira de tomate tipo cereja. Jornada Integrada em Biologia. p. 67-73. 2016. Disponível em: <http:// editora.unoesc.edu.br/index.php/ jornadaintegradaembiologia/article/view/10202/ 5576>. Acesso em: nov.2018

KADER, A.A. Postharvest technology of horticultural crops. 3.ed. California: University of California, 2002. 535 p.

KLUGE, R.A.; MINAMI, K. Efeito de ésteres de sacarose no armazenamento de tomates 'Santa Clara'. Scientia Agrícola, v.54, n.1-2, p.39-44, 1997.

KORNACKI, J.L.; JOHNSIN, J.L.

Enterobacteriaceae, coliforms, and Escherichia coli as quality and safety indicators. In: DOWNES, F.P; ITO, K. (Ed.). Compendium of methods for the microbiological examination of foods. 4. ed. Washington, DC: American Public Health Association - APHA, 2001. Washington, 2001.p. 69-82. 
KORTE, K.P.; FAVARÃO, S.C.M. Efeito da gelatina incolor e comercial associada a extratos vegetais como revestimento comestível na pós-colheita do morango. Revista Ciências Exatas e da Terra e Ciências Agrárias, v.1, p.8-15, 2016.

LEMOS, O.L.; REBOUÇAS, T.N.H.; JOSÉ. A.R.S.; VILA, M.T.R; SILVA, K.S. Utilização de biofilme comestível na conservação de pimentão 'Magali r' em duas condições de armazenamento.

Bragantia, v.66, n.4, p.693-699, 2007.

LIMA, G.S.; SANTOS, M.J.P.; ANDRADE, R.O.; SILVA, J.G.; SOUZA, S. Uso de revestimentos comestíveis pós-colheita de pimentão verde armazenado em temperatura ambiente e sob refrigeração. Caderno Verde de Agroecologia e Desenvolvimento Sustentável, v.4, p.1-6, 2014.

MARQUES, L.C.S.; FINGER, F.L.; CORDEIRO, D.C.; FOGAÇA, C.M. Sensibilidade de pimentas a injuria por frio. Horticultura Brasileira (Suplemento), 23:447, 2005.

MATTIUZ, B.H.; MIGUEL, A.C.A.; GALATI, V.C.U.; NACHTIGAL, J.A.C. Efeito da temperatura no armazenamento de uvas apirênicas

minimamente processadas. Revista Brasileira Fruticultura, v.31, n.1, p.44-052, 2009.

MORGADO, C.M.A.; DURIGAN, J.F.; SANCHES, J.; GALATI, V.C.; OGASSAVARA, F.O. Conservação pós-colheita de frutos de pimentão sob diferentes condições de armazenamento e filmes.

Horticultura Brasileira, v.26, n.2, 2008.

OLIVEIRA, M.A.; CEREDA, M.P. Pós colheita de pêssego revestido com filme a base de amido como alternativa a cera comercial. Brazilian Journal of Food Tecnology, v.23, p.28-33, 2003.

OLIVEIRA, G.G.H.; CORRÊA, P.C.; BAPTESTINI, F.M.; FREITAS, R.L.; VASCONCELLOS, D.S.L. Controle do amadurecimento de goiabas "Pedro Sato" tratadas por frio. Enciclopedia

Biosfera, v.6, p.1-15, 2010.
OLIVEIRA, E.N.A.; SANTOS, D.C. Tecnologia e processamento de frutos e hortaliças. Natal: IFRN, 2015. 234 p.

PINHEIRO, N.M.S. Revestimentos com cera de carnaúba incorporados de antimicrobianos em caju (Anacardium Occidentale L) e goiaba (Psidium Guajava). 2012. 125 f. Tese (Doutorado em Ciência e Tecnologia de Alimentos) - Universidade Federal de Viçosa, Viçosa, 2012.

PINTO, C. M. F.; PINTO, C. L. O.; SOUSA, S.; DONZELES, S. M. L.; SOARES; GAIOTTO, M. C. Conservação pós-colheita de Pimenta Dedo-demoça com Revestimento de Cera de Carnaúba. In: I Congresso Brasileiro de Processamento de Frutas e Hortaliças, 2009. I Congresso Brasileiro de Processamento de Frutas e Hortaliças.

REIS, K.C.; ELIAS, H.H.S.; LIMA, L.C.O.; SILVA, J.D.; PEREIRA, J. Pepino japonês (Cucumis sativus L.) submetido ao tratamento com fécula de mandioca. Ciência Agrotécnica, v. 30, p. 487-493, 2006.

SCANAVACA, L. J.; FONSECA, N.; PEREIRA, M. E. C. Uso de fécula de mandioca na pós-colheita de manga 'surpresa'. Revista Brasileira de Fruticultura, v.29, p.67-71, 2007.

VALVERDE, R. M.V. Composição bromatológica da pimenta malagueta in natura e processada em conserva. 2011. 54f. Dissertação (Mestrado). Engenharia de Processos de Alimentos Itapetinga - BA: UESB, 2011.

VICENTININ, N.M.; CEREDA, M.P.; CAMARA, F.L.A. Revestimento de fécula de mandioca, perda de massa e alteração da cor de frutos de pimentão. Scientia Agricola, v.56, n.3. p.713$716,1999$.

ZENEBON, O.; PASCUET, N.S.; TIGLEA, P. Métodos físico-químicos para análise de alimentos. São Paulo: Instituto Adolfo Lutz. 4. ed. 2008. 1020 p.

Recebido para publicação em 24/9/2018 e aprovado em 30/12/2018. 